\title{
SEGMENTAÇÃO A PARTIR DAS BASES ESPECÍFICAS NÃO OBSERVÁVEIS DO PRODUTO E O EMPREGO CONJUNTO DE TÉCNICAS MULTIVARIADAS: $O$ ESTUDO DE CASO DO VEÍCULO MULTIUSO
}

\author{
Solon Bevilacqua \\ solbev@gmail.com \\ Universidade Federal de Goiás - Goiânia, GO / Brasil
}

Recebido em 23//10/2012

Aprovado em 05/03/2013

Disponibilizado em 01/08/2013

Avaliado pelo sistema double blind review

Revista Eletrônica de Administração

Editor: Luís Felipe Nascimento

ISSN 1413-2311 (versão on-line)

Editada pela Escola de Administração da Universidade Federal do Rio Grande do Sul

Periodicidade: Quadrimestral

Sistema requerido: Adobe Acrobat Reader.

\section{RESUMO}

Este estudo exploratório e descritivo pesquisou uma base de dados primários, potenciais consumidores de um veículo multiuso, com posterior emprego de Análise Conjunta (CA) e Análise de Conglomerado (AC), conforme proposta de Green e Krieger (1991). A abrangência da pesquisa teve caráter nacional, em que se investigou o emprego das utilidades parciais para conforto, capacidade de carga e preço como recursos de entrada para uma base de segmentação, constatando-se um alinhamento entre os métodos e coerência entre os resultados obtidos. Os ganhos observados nesse estudo estão relacionados a três aspectos: [1] execução da pesquisa: partindo de um questionário complexo e aplicado para a diversificada população alvo brasileira, obteve-se êxito na pesquisa nessas condições singulares; [2] Manipulação das variáveis: contando com variáveis não observáveis - de difícil manipulação em contraste com as empregadas no estudo original - obtendo êxito sem perdas observadas; [3] Validação: empregando o método proposto, ambas as técnicas (CA e AC) apresentaram resultados semelhantes, o que confere precisão ao método sugerido. A geração de resultados similares, a partir do emprego conjunto de ambas as técnicas, sugere alinhamento metodológico e validação da teoria. Considera-se ao final do estudo que empregar metodologia de identificação de atributos voltada para a segmentação de mercado pode atenuar a questão de reposicionamento do conceito. Como limitação do estudo destaca-se a questão da falta de estudos correlatos, em outras áreas que efetivamente coloquem em prática a robustez da técnica. Sugestiona-se para trabalhos futuros, um estudo de maior amplitude em situação comprobatória. Como principal contribuição do estudo, constatou-se a facilidade de replicar os estudos dos autores.

Palavras-Chave: Segmentação de Mercado; Atributos, Análise Multivariada; Pesquisa; Parth Worth's. 
Segmentação a partir das bases específicas não observáveis do produto e o emprego conjunto de técnicas multivariadas: o estudo de caso do veículo multiuso

\title{
SEGMENTATION FROM SPECIFIC NON OBSERVABLE BASIS ON THE PRODUCT AND THE COMBINED USE OF MULTIVARIATE TECHNIQUES: MULTIUSE VEHICLE CASE STUDY
}

\begin{abstract}
This exploratory and descriptive study seeked a base of primary data, potential consumers of a multiuse vehicle, with further use of Conjoint Analysis and Cluster Analysis, according to Green \& Krieger's proposal (1991). The scope of the study was local, where was investigated the use of partial uses for comfort, loading capacity and price as input resource to a segmentation basis. There was an alignment between the methods and consistency between the results. The gains observed in this study are related to three aspects: [1] research execution: starting from a complex questionnaire applied for a Brazilian diversified target population, obtaining success in this research singular conditions; [2] data manipulation: considering unobservable variables - difficult to handle in comparison with those used in the original study - it was obtained success without observed losses; [3] Validation: using the proposal method, both techniques (AC and $\mathrm{CA}$ ) presented similar results, which ensures precision to the suggested method. The generation of similar results, from the combined use of both techniques, suggests methodological alignment and theory validation. For so it's possible to consider that applying this methodology of attributes identification focused on market segmentation, can mitigate this repositioning concept question. The lack of correlated studies question, in other segments, that actually practice this technical hardiness, is highlighted as a study limitation. We suggest as future studies, a best evidentiary situation capability application. The main contribution of this study was to show how easy is to replicate the authors studies.
\end{abstract}

Keywords: Market segmentation; Attributes; Multivariate analysis; Research; Part Worth's.

\section{INTRODUÇÃO}

Os primeiros estudos sobre segmentação remontam à década de 1930, a partir das pesquisas relacionadas à Teoria da Concorrência Imperfeita. Um de seus pressupostos era que o consumidor não poderia ter suas necessidades e características heterogêneas desprezadas quando da oferta de um produto ou serviço. Dessa forma, conforme Robinson (1938) e Smith (1956), um mercado heterogêneo deveria ser visto como uma série de mercados homogêneos com várias preferências e inúmeros segmentos distintos.

A seguir, buscou-se um procedimento de segmentação que considerasse o mix de marketing, relacionando variáveis como tipos de produtos, níveis de preço, comunicações para cada público e formas de entrega dos produtos. Com o aprimoramento das técnicas estatísticas,

REAd I Porto Alegre - Edição 75 - N 2 - maio/agosto 2013 - p. 433-452 
agregou-se relação entre pessoas e o mix de marketing. Posteriormente, as variáveis sóciodemográficas e econômicas foram introduzidas para descrever as diferenças de consumidores, tendo, atualmente, fraca contribuição, dada a complexidade observada nas práticas de consumo.

A partir da constatação de que havia competição entre microambientes com produtos substitutos, surgiu a necessidade de agregar bases específicas não observáveis (benefícios, elasticidade, preferências, intenções e atributos), e saber utilizá-las no processo de segmentação, condição sine qua non para o êxito da estratégia mercadológica.

Este estudo objetiva validar a teoria de segmentação de mercado de Green e Krieger (1991) por meio de um estudo de caso, empregando sequencialmente a Análise Conjunta e Análise de Conglomerados. São objetivos específicos do estudo: [1] realizar um estudo exploratório e descritivo junto a potenciais consumidores do veículo multiuso; [2] empregar as utilidades parciais obtidas a partir da Análise Conjunta de Atributos na Análise de Conglomerados; [3] comparar e analisar as possibilidades que o emprego das duas técnicas pode gerar para as decisões de segmentação.

O tópico a seguir reserva uma apresentação das principais questões que cercam os atributos do produto, sequencialmente, o processo de compra/avaliação das alternativas e o julgamento cognitivo. O método exploratório e descritivo que emprega duas técnicas multivariadas em conjunto é o assunto da segunda seção. Nesse tópico, discutir-se-á o esquema de desenvolvimento da pesquisa, manipulação das variáveis e os aspectos teóricos sobre as técnicas. As análises, discussão dos resultados e principais conclusões encerram este estudo.

\section{REFERENCIAL TEÓRICO}

\subsection{Reflexões sobre os atributos de consumo}

Comumente, o conceito de benefício é confundido com o de atributo. Segundo Markin, Narayana (1975); Wu, Day e MacKay (1988) e Mowen e Minor (2008), os benefícios são domínios distintos de utilidades, sendo considerados no momento da escolha entre alternativas. Seriam resultados positivos para o consumidor e apresentam as características físicas e concretas de um produto ou serviço.

Alpert (1971) criou a classificação de benefícios em salientes, importantes e determinantes. Os primeiros seriam aqueles tidos como sem importância na decisão de compra, mas de fácil REAd I Porto Alegre - Edição 75 - N 2 - maio/agosto 2013 - p. 433-452 
Segmentação a partir das bases específicas não observáveis do produto e o emprego conjunto de técnicas multivariadas: o estudo de caso do veículo multiuso

percepção. Os tidos como importantes são aqueles considerados não decisores de compra, mas que possuem relativa importância. Finalmente, os determinantes seriam aqueles capazes de influenciar a compra de um produto ou serviço.

Os atributos de um produto representam o maior estímulo que influencia o consumidor em sua tomada de decisão de compra. Segundo Peter e Olson (1996), há o pressuposto de que pesariam em sua decisão aspectos associados a valores, a crenças ou a experiências passadas. Tais consequências agregariam uma divisão dentre os atributos, advindos de resultados da experiência direta de consumo de um produto. No dizer de Ratchford (1982), as consequências, se positivas, são os benefícios que a compra ou o consumo de determinado produto acarretam.

A partir da década de 1990, ocorreu uma profusão de classificações para os atributos, dada a sua importância crescente nos estudos de mercado. Nowlis e Simonson (1997) apresentam a divisão dos atributos em comparáveis e enriquecidos. Os primeiros seriam aqueles que são de fácil avaliação entre as alternativas de compra e os tidos como enriquecidos são mais significativos quando analisados separadamente do produto.

Woodruff e Gardial (1996) afirmam que os atributos podem ser hierarquizados num contexto de valor, que conecta as características do produto às características psicológicas do consumidor. São os primeiros a ser percebidos pelos consumidores, seguidos dos atributos abstratos e das consequências funcionais e psicológicas.

Para Assael (1998), o estudo e a mensuração dos atributos são fundamentais ao entendimento das crenças do consumidor a respeito de um determinado produto ou marca. Entretanto, as crenças que cercam os atributos de um produto podem distorcer a realidade de consumo. Mowen e Minor (2008) tratam dessa questão, exemplificando com o efeito de halo, que causa percepções equivocadas, um fenômeno de generalização para as características de um produto ou serviço. A característica recebe uma carga de atenção diferenciada de cada consumidor e esse aspecto impacta diretamente a importância dada ao atributo. Em ações de Marketing específicas, essas características podem ser ressaltadas tendo a atenção do consumidor direcionada a elas, aumentando a importância percebida.

Para Allen (2001), os atributos podem ser tangíveis ou objetivos, os últimos perceptíveis por meio dos cinco sentidos humanos, ao passo que intangíveis seriam as imagens do produto tais como beleza, estética ou prestígio. Conforme o autor, os atributos são julgados distintamente pelos consumidores, sendo necessária cautela na análise. O autor argumenta que, até os REAd I Porto Alegre - Edição 75 - N² 2 - maio/agosto 2013 - p. 433-452 
estudos de Henke (1987), dava-se mais enfoque aos aspectos tangíveis (atitudes avaliativas), em detrimento dos aspectos intangíveis (atitudes expressivas). Assim, propuseram uma visão ampliada para o entendimento das atitudes dos consumidores, identificando o impacto causado no comportamento do consumidor. Os valores humanos podem ser incluídos à tradicional visão de atitudes, classificando os valores humanos em instrumentais (como crença em liberdade, e uma vida com conforto) e terminais (como crença em independência, ambição e honestidade). Também resgatam a visão das atitudes, dos valores humanos, criando, assim, um sistema de valor, atitude e comportamento, subdividido em funções instrumentais e expressivas.

A valorização dos atributos pelo consumidor começa a receber importância no final da década de 1980. Holbrook (1987) cria uma classificação denominada Attitude Function Theory, dividida em instrumental ou função expressiva, disposta a entender as atitudes e valorização de atributos dos consumidores. Posteriormente, a classificação foi discutida por autores como Allen e Wison (2002); Shavitt (1990); Snyder e DeBono (1985) e Spivey, Munson e Locander (1983).

A função de atitude pode ser dividida em dois grupos, conforme Henke (1987); seriam eles: 'grupo avaliativos' e 'grupo expressivos'. O primeiro grupo se refere à interação entre objeto e pessoa, resultando em recompensas ou privações tangíveis e quantificáveis. A segunda classificação diz respeito não somente à relação entre pessoa e objeto, mas, também, de questões psicológicas que buscam incrementar a autoestima, suas recompensas ou privações. Dessa forma, as atitudes expressivas poderiam ser divididas em sociais, valores expressivos e atitudes defensivas.

Essa preocupação em classificar os atributos e as atitudes se estende para o estudo do processo de compra e avaliação das alternativas. A questão ganha expressividade não somente com a popularização dos softwares estatísticos, mas também com a disseminação do estudo para outras áreas.

\subsection{Processo de compra e avaliação das alternativas}

Esse processo pode ser suportado por duas teorias distintas — a teoria cognitiva da aprendizagem e a teoria da opinião social. A partir da primeira teoria, conforme Lutz (1991), os benefícios são crenças formadas por processos de aprendizagem, com o objetivo de sanar um problema. Considerando a teoria da opinião social, segundo Herrmann (1994), os REAd I Porto Alegre - Edição 75 - N 2 - maio/agosto 2013 - p. 433-452 
Segmentação a partir das bases específicas não observáveis do produto e o emprego conjunto de técnicas multivariadas: o estudo de caso do veículo multiuso

benefícios seriam mecanismos que funcionariam como filtros de estímulos externos e que, em conjunto com a formação de preferências, possibilitariam a passagem dos estímulos a uma resposta. Tendo as duas teorias como eixo, seria possível explicar os comportamentos a partir dos mecanismos psicológicos internos que dão uma resposta a uma necessidade.

A partir de Kanuk e Schiffman (1997), surgem três tipos de comportamento de compra: aqueles tidos como experimentais, repetidos, e os de longo prazo. Tal classificação sugere um processo de compra que sintetize a tomada de decisão. Esse processo, subjacente à teoria cognitiva, comumente é representado por cinco fases distintas. Conforme Sheth, Mittal \& Newman (2001); Mowen e Minor (2008); Blackwell, Miniard e Engel (2008), as fases seriam o reconhecimento do problema, a busca por informação, a avaliação de alternativas, a compra e finalmente a experiência pós-compra. No caso específico desse estudo, a fase de Avaliação seria a mais importante, detalhada a seguir.

Essa perspectiva tradicional da tomada de decisão oferece ênfase para uma abordagem racional do processamento de informação para a ação de compra. Conforme Mowen e Minor (2008), ela está bastante associada à abordagem da hierarquia de efeitos de alto envolvimento, associadas à formação de atitudes.

Conforme o autor, há cerca de três décadas, esse modelo linear com altos níveis de processamento de informação cede espaço para uma teoria em que os consumidores nem sempre agem sob um processo estendido de decisão. Trata-se da perspectiva experimental e, nesse caso, o estágio de avaliação das alternativas praticamente é extinto no processo de decisão, considerando que um processo simplificado está em andamento. Sob essa ótica, o consumidor é tido como aquele que consome a partir de sensações, sentimentos, imagens e emoções que os produtos despertam.

A perspectiva experimental é confrontada com uma controversa teoria, conhecida como perspectiva da influência comportamental. Conforme Mowen e Minor (2008) as contingências são capazes de influenciar o comportamento dos consumidores (luminosidade, a disposição de produtos na loja, texturas, odores, entre outros).

Ao dar início no processo de avaliação das alternativas, o consumidor inicia uma fase no processo decisório que, se considerada satisfatória, trará impacto significativo para o sucesso da compra. Conforme Blackwell, Miniard e Engel (2008), os consumidores não podem construir conjuntos de opções baseados na busca interna da memória sem um conhecimento prévio de algumas alternativas.

REAd I Porto Alegre - Edição 75 - N 2 - maio/agosto 2013 - p. 433-452 
Considerando um processo de baixo envolvimento, segundo Mowen e Minor (2008), a avaliação das alternativas consiste na formação de algumas crenças rudimentares acerca das opções disponíveis. Para os autores, na perspectiva da influência comportamental, os consumidores nunca comparam conscientemente as alternativas e descobrem até que ponto as opções contam com vários atributos. Se considerado um atributo de expressivo esforço cognitivo na escolha, pode ocorrer um processo de abandono para a opção.

\subsection{Julgamento cognitivo}

São vários os modelos para comparar produtos e seus respectivos atributos, recebendo destaque o Compensatório Linear Simultâneo e o Não Compensatório Sequencial. O primeiro modelo é fundamentado em crenças sobre os atributos e avaliações. Enquanto um atributo recebe escores baixos, outro adquire escore maior. Dessa forma, os atributos são multiplicados pelas avaliações que os indivíduos fazem de cada um deles e uma crença total do objeto é produzida ao somar-se cada um dos atributos. Fishbein (1967) propõe um dos principais modelos, tendo um conjunto de crenças de um determinado comportamento multiplicado pelas avaliações de todas as crenças, somadas às normas subjetivas e multiplicado pela motivação para se comportar a essas normas, leva a um indicador, intenção comportamental.

Nesse modelo compensatório, conforme Sheth, Mittal e Newman (2001), o consumidor chega a determinada opção escolhendo entre todas as alternativas de atributos disponíveis, compensando pontos fracos e fortes. Trata-se de um raciocínio algébrico, algumas vezes ponderado, em que o consumidor maximizaria os atributos positivos.

No Modelo Não Compensatório Sequencial, um atributo não é compensado por escores maiores em outros atributos. Conforme Wright (1975), sua fundamentação é a de que questões indesejáveis do produto não devem ser compensadas por questões desejadas e os indivíduos utilizam decisões sequenciais para considerar atributos. Uma hierarquia de avaliação é utilizada na escolha e as alternativas vão sendo eliminadas à medida que a avaliação ocorre.

Nessa modalidade de julgamento o consumidor determina os limites mínimos de todos os atributos importantes e aquelas que atenderem a essa exigência podem ser escolhidas. Segundo os autores, se todas as opções não corresponderem, podem ser abandonadas para consequente revisão de níveis mínimos ou mesmo para busca de novo modelo decisório.

REAd I Porto Alegre - Edição 75 - Nº 2 - maio/agosto 2013 - p. 433-452 
Segmentação a partir das bases específicas não observáveis do produto e o emprego conjunto de técnicas multivariadas: o estudo de caso do veículo multiuso

Sheth, Mittal e Newman (2001) apresentam uma opção de julgamento denominada Modelo Não Compensatório Disjuntivo, que implica compensar entre os aspectos das alternativas de escolha. Um atributo de um determinado produto serviria para amenizar a fraqueza de outro. O modelo de Eliminação por Aspectos corresponde à atividade de classificação dos atributos em ordem de importância, além de definir valores de prioridade. O modelo Lexicográfico assemelha-se ao anterior, diferindo apenas na questão de não eliminação de aspectos dos atributos.

A partir de Sheth, Mittal e Newman (2001); Mowen e Minor (2008) e Miniard e Engel (2008), tem-se uma variante para os modelos não compensatórios: (conjuntivos, disjuntivos, lexicográfico e de eliminação por aspectos) Nessa classificação, recai um emprego para produtos de baixa elasticidade e para estratégias rotineiras e limitadas. Também podem ser usados na fase inicial de uma resolução de problemas. Os autores também afirmam que os modelos podem ser empregados conjuntamente, primeiramente um não compensatório e, posteriormente, um compensatório, para identificação ótima da escolha. Tal processo é denominado estratégia de decisão de duas fases.

\section{MÉTODO DA PESQUISA}

A primeira etapa deste estudo, de caráter exploratório e descritivo, consistiu em criar as variáveis para identificação dos part worth's, que posteriormente serão empregados na Análise de Conglomerados. Para tanto, segundo metodologia consagrada por Morgan (1988), obteve-se a partir de uma base de dados secundários, um cadastro de antigos proprietários de automóveis, que, historicamente, eram potenciais compradores de veículos Multiuso. Esse mesmo estudo identifica que, comumente, o consumidor, adquire um veículo multiuso em função de três atributos (1) Conforto Interno, (2) Capacidade de Carga/Bagagem e finalmente, pelo (3) Preço.

O veículo multiuso foi lançado em 2001, tendo como concorrentes diretos o Kangoo (Renault), Partner (Peugeot) e Berlingo (Citröen). Seus donos, em geral, são pais com dois ou três filhos pequenos, que transportam muito volume ou então quem viaja muito e necessita de espaço para bicicletas e material de camping. O projeto do porta-malas com 602 litros e a porta lateral corrediça são considerados diferenciais no modelo.

REAd I Porto Alegre - Edição 75 - N 2 - maio/agosto 2013 - p. 433-452 
O conceito de veículo multiuso foi idealizado para atender a duas demandas com um só produto. Conforme AN Veículos (2009), na Europa, é muito comum o emprego de um veículo para atendimentos rápidos de entregas ou pequenos consertos durante a semana e, nos finais de semana, o mesmo veículo ser utilizado para deslocamentos de lazer para a família. Dessa forma, idealizou-se um automóvel que conjugasse conforto, capacidade de carga e um preço atraente para esse profissional e sua família.

A amostra foi constituída de 810 potenciais compradores, que receberam o questionário por correio, tendo essa opção de reenvio ou ainda por meio de um site disponibilizado na Internet. A origem e a natureza do banco de dados não serão detalhadas, por questões de confidencialidade.

Os questionários empregados para a coleta de dados de um estudo que emprega Análise Conjunta de Atributos são tidos como os mais complexos existentes, em função da dificuldade da entrevista. Os trade off's para a identificação das cestas de preferências tendem a confundir o entrevistado, acarretando incremento do erro da pesquisa. Objetivando minimizar esse problema, criaram-se "âncoras" para definir o que seria baixo, médio e alto conforto e baixa, média e alta capacidade de bagagem, construindo níveis de atributos para as três variáveis empregadas. Ao todo, os questionários foram compostos por 27 trade off's $(3 * 3 * 3=27)$, com posterior exclusão de dois perfis considerados impraticáveis ou incoerentes (Capacidade de Carga Alta, Conforto Alto e Preço de 26.000,00; Capacidade de Carga Baixa, Conforto Baixo e Preço de 29.500,00). A variável dependente é a nota, que possui modalidade de resposta variando de 1 a 10. O Quadro 1 apresenta a classificação dos atributos.

\begin{tabular}{|c|c|}
\multicolumn{2}{|c}{ Quadro1 - Importância e Utilidade } \\
\hline Atributo & Nível do Atributo \\
\hline \multirow{2}{*}{ Capacidade de Carga } & Alta \\
\cline { 2 - 2 } & Média \\
\cline { 2 - 2 } & Baixa \\
\hline \multirow{2}{*}{ Conforto } & Alta \\
\cline { 2 - 2 } & Média \\
\cline { 2 - 2 } & Baixa \\
\hline \multirow{2}{*}{ Preço } & $26.000,00$ \\
\cline { 2 - 2 } & $28.500,00$ \\
\cline { 2 - 2 } & $29.500,00$ \\
\hline
\end{tabular}

Fonte: Elaborado pelo autor

Com relação à Análise de Conglomerados, a medida de distância escolhida foi a euclidiana e o processo de aglomeração, o método não hierárquico $K$-Means. Apesar de ter a desvantagem REAd I Porto Alegre - Edição 75 - Nº 2 - maio/agosto 2013 - p. 433-452 
Segmentação a partir das bases específicas não observáveis do produto e o emprego conjunto de técnicas multivariadas: o estudo de caso do veículo multiuso

de se ter de definir o número de conglomerados, esse método foi adotado, em virtude da clarividência de já se ter o domínio da informação da quantidade de clusters (3). Essa escolha também foi priorizada em virtude da técnica de agrupamento não hierárquico "ser relativamente instável e pouco confiável" conforme Aaker, Kumar e Day (2001). Outra força desse teste é a praticidade de já se ter os centros finais de aglomeração que representam as médias das variáveis para os casos nos conglomerados finais. Hair, Anderson, Tathan e Black (2005) indicam uma segunda forma de traçar o perfil dos entrevistados, ignorando variáveis que foram excluídas do processo de aglomeração, essas variáveis seriam demográficas ou psicográficas. Com relação à questão de confiabilidade, duas providências podem ser tomadas, de fácil emprego, adotadas nesse artigo: a primeira seria separar o grupo de dados em duas metades, e comparar os centróides dos clusters nas duas subamostras e a segunda seria ir realizando repetições múltiplas até a estabilização da solução.

A fidedignidade do estudo foi obtida por meio do nível de Stress (Kruskal, 1964) definindo-se quão ruim seria a diferença entre as medidas de proximidade e as correspondentes distâncias. Borg e Groenen (1997) afirmam que o nível de Stress varia de acordo com alguns fatores, tais como número de pontos (quanto mais pontos, mais Stress) e dimensionalidade (maior dimensionalidade, menor Stress).

Por meio do Diagrama de Shepard, buscou-se, também, outra forma de avaliar a previsão, plotando-se as distâncias reproduzidas contra as distâncias observadas. Em ambos os modelos foi constatado que a pesquisa estava conforme. O índice de Stress encontrado foi de 0,000010, tido como excelente e o diagrama de Shepard apresentou ajuste de dados ao modelo.

\subsection{Desempenho das técnicas}

A Análise Conjunta de Atributos (AC) ou Conjoint Analysis tem registrado expressiva aceitação e utilização. Segundo Aaker, Kumar e Day (2004) já na década de 1980 mais de 400 análises conjuntas para fins comerciais eram realizadas anualmente. Conforme o autor, as aplicações para a técnica que recebem destaque seriam:

- Em análises em que os produtos ou serviços possuem um número de atributos, cada qual com dois ou mais níveis;

- Onde não existam ainda combinações possíveis entre os níveis de atributos;

- Onde possa existir expansão dos níveis dos atributos para além daqueles disponíveis na ocasião;

REAd I Porto Alegre - Edição 75 - N 2 - maio/agosto 2013 - p. 433-452 
- Onde a tendência geral seja provável e já conhecida sobre os atributos

A Análise Conjunta é adequada para algumas formas de segmentação. Sua aplicação principal recai diretamente sobre a medição de preferências do comprador para os níveis de atributo de produto e, conforme Green e Krieger (1991), nos benefícios que o comprador possa obter a partir dos atributos desse consumo.

Segundo os autores, existem algumas tendências recentes que têm servido para tornar o método ainda mais aplicável à segmentação do mercado. Os softwares disponíveis são amigáveis e relativamente baratos, além de serem providos de interface para o trabalho com Análise de Agrupamentos.

Como a Análise Conjunta revela dados de utilidade a partir de amostra selecionada, segundo Motta (1987, p. 22), é possível estudar as discrepâncias de utilidade ao longo dessa amostra, "procurando identificar grupos distintos de consumidores, sendo útil para análise de segmentação”. Poder-se-ia submeter as utilidades obtidas em cada nível, a um programa de aglutinação dos entrevistados, a partir das respostas fornecidas individualmente.

Adicionalmente, Bittencourt e Fachel (1999), afirmam que por meio de combinações que representam conjuntos de escolhas reais, a técnica proporciona a identificação de configurações ótimas de produtos ou serviços, que esclarecem potencial de novas oportunidades de mercado ou potencial de consumo. Portanto, o objetivo da técnica é determinar as contribuições das variáveis preditoras e de seus respectivos níveis, para construir um modelo consistente de preferências de consumo.

A Análise Conjunta pode também ser útil, quando houver interesse em avaliar as percepções dos consumidores em relação a diversos estímulos pertencentes a um mesmo propósito. Conforme Motta (1987) as utilidades presentes em cada estímulo confere ganhos frente ao método tradicional decompositivo, afinal o consumidor se posiciona frente a uma cesta de opções e não para utilidades isoladas. Reagindo a estímulos apresentados pela combinação de níveis de atributos, o consumidor dá margem para que se avance até sua estrutura mental de compensação, provendo informações valiosas sobre seu sistema de valores e de avaliação de preferências.

Com relação à Análise de Conglomerado, Malhotra (2001) aborda a técnica sugerindo seu emprego para a definição da relação de interdependência entre o conjunto de variáveis. Segundo o autor, as aplicações mais clássicas envolvem segmentação de mercado, compreensão do comportamento do consumidor e identificação das oportunidades de um REAd I Porto Alegre - Edição 75 - N 2 - maio/agosto 2013 - p. 433-452 
Segmentação a partir das bases específicas não observáveis do produto e o emprego conjunto de técnicas multivariadas: o estudo de caso do veículo multiuso

novo produto. Conforme Fávero, Belfiore, da Silva e Chan (2009), a variável estatística de agrupamento se define como o conjunto de atributos das observações que servirão de base para a determinação da similaridade entre elas. Essa base gerada a partir de atributos torna-se mais robusta, se proveniente de uma técnica que privilegie o emprego desses dados de entrada, como é o caso da Análise Conjunta. Para Brochado e Martins (2008) os métodos hierárquicos permitem recuperar a heterogeneidade existente nos dados e a obtenção simultânea de estimativas ao nível do individuo.

Alguns problemas associados ao emprego isolada da Análise de Conglomerados são conhecidos e detalhados em Aldenderfer e Blashfield (1984) e Everitt (1980). Destaca-se a questão da validade dos grupamentos, se efetivamente são genuínos, conferindo imprecisão para o estudo mercadológico. Utilizar a Análise Fatorial previamente não resolveria essa fragilidade, considerando que, segundo Aldenderfer e Blashfield (1984), poderia ocorrer uma perda de dados relevantes acarretando perda de informação para a Análise de Conglomerados. Essa problemática não se apresenta no emprego preliminar da Análise Conjunta.

Considerando a metodologia original de Green e Krieger (1991) e as limitações encontradas pelos autores, tem-se que a ferramenta é tida como um recurso de planejamento e de análise de sensibilidade para explorar alternativas de produtos e estratégias de preços. A técnica não foi testada para atributos intangíveis como conforto, e muita pesquisa adicional será necessária antes que o seu potencial seja comprovado.

\section{ANÁLISES DOS DADOS E RESULTADOS}

A amostra do estudo foi constituída por pessoas com idade mínima de 25 anos e máxima de 46 , sendo que a principal distribuição concentrou-se entre 35 a 42 anos $(49,66 \%)$. Do total de 810 respondentes, $64,14 \%$ eram do sexo masculino e $38,86 \%$, do sexo feminino, dispostos em famílias com mais de quatro integrantes $(69,66 \%$ da amostra possuíam famílias tendo entre quatro a seis indivíduos). Buscou-se esse perfil em função da apresentação dos dados secundários, que previam esse perfil para o comportamento de compra do automóvel multiuso, homens com mais de 35 anos com famílias tidas como grandes.

Conforme Aaker, Kumar e Day (2004, p. 615), os valores para cada atributo em seus níveis, ou part worth utilities devem ser colocados de forma decrescente e, dessa forma, diminuídos REAd I Porto Alegre - Edição 75 - N 2 - maio/agosto 2013 - p. 433-452 
um a um para obter a menor e a maior diferença. "Quanto maior a diferença entre o valor mais alto e o mais baixo dos níveis de um atributo, maior sua importância. Ao contrário, se todos os níveis tiverem a mesma utilidade, o atributo não é importante [...] não exerce atitude gerencial". A tabela 1 apresenta a Diferença entre Part Worth's.

Tabela 1 - Diferença entre Part Worth's

\begin{tabular}{l|lc}
\hline Atributos & Níveis dos Atributos & Part Worth's \\
\hline \multirow{4}{*}{ Capacidade } & Capacidade de Carga Baixa & 0,429 \\
& Capacidade de Carga Alta & 0,257 \\
& Capacidade de Carga Média & 0,193 \\
& Diferença entre o maior e menor valor & $\underline{0,236}$ \\
Conforto & Conforto Alto & 0,564 \\
& Conforto Baixo & 0,559 \\
& Conforto Médio & 0,116 \\
Preço & Diferença entre o maior e menor valor & $\underline{0,448}$ \\
& Preço Médio & 0,187 \\
& Preço Baixo & 0,151 \\
& Preço Alto & 0,106 \\
& Diferença entre o maior e menor valor & $\underline{0,081}$ \\
\hline
\end{tabular}

Fonte: Elaborado pelo autor

A diferença entre os maiores e menores valores sugere que o Conforto seja o atributo mais significativo para o consumidor, seguido pela Capacidade de Carga e em terceiro lugar, o Preço.

O emprego da Análise Conjunta de Atributos, por meio da soma dos pesos de importância relativa (total worth's), revela que o nível conforto é o atributo de maior importância, com $43,95 \%$ do total da preferência declarada, seguido por nível de capacidade de carga, 41,71\%, e finalmente por preço, $14,34 \%$. Os procedimentos de cálculo obedecem à soma das utilidades parciais relativas vistas anteriormente, divididas pelo total da utilidade do modelo. O Quadro 2 apresenta os dados relativos à importância e à utilidade do produto.

REAd I Porto Alegre - Edição 75 - N 2 - maio/agosto 2013 - p. 433-452 
Segmentação a partir das bases específicas não observáveis do produto e o emprego conjunto de técnicas multivariadas: o estudo de caso do veículo multiuso

Quadro 2 - Importância e Utilidade

\begin{tabular}{|c|c|c|c|}
\hline Atributo & Nível & Part Worth's & Importância Relativa \\
\hline \multirow{3}{*}{ Conforto } & Baixo & $-0,559$ & \multirow{3}{*}{$43,95 \%$} \\
\hline & Médio & $-0,116$ & \\
\hline & Alto & $-0,564$ & \\
\hline \multirow{3}{*}{ Capacidade de Carga } & Baixo & $-0,429$ & \multirow{3}{*}{$41,71 \%$} \\
\hline & Médio & $-0,193$ & \\
\hline & Alto & $-0,257$ & \\
\hline \multirow{3}{*}{ Preço } & $26.500,00$ & $-0,151$ & \multirow{3}{*}{$14,34 \%$} \\
\hline & $28.000,00$ & $-0,187$ & \\
\hline & $29.500,00$ & $-0,106$ & \\
\hline
\end{tabular}

Fonte: Elaborado pelo autor

A composição desses três grupos é premissa básica para a continuidade das análises, que agora envolverão o emprego da Análise de Conglomerados, objetivando a definição dos grupos de consumidores com perfis comuns. O pré-requisito para essa estrutura é aplicar a análise anterior para cada um dos 810 casos, e trabalhar com os três níveis de valores parciais, que, somados, resultam nos parth worth's. Optou-se por adotar como medida de semelhança, a distância euclidiana e o processo de aglomeração não hierárquica, K-Means Clustering, afinal é premissa para o emprego desse tipo de método, o pré-estabelecimento do número de conglomerados, e a própria Análise Conjunta de Atributos já indicou, os mesmos três grupos anteriores. A análise de elbow criterium também apresentou esses resultados. A Análise de Conglomerado fixou três grupos distintos, denominados a posteriori (1) Trabalhadores - 286 casos. (2) Requintados - 325 casos e (3) Populares - 189 casos. A Tabela 2 apresenta a análise dos agrupamentos. 
Solon Bevilacqua

Tabela 2 - Análise de Agrupamentos

\begin{tabular}{lc}
\hline \multicolumn{1}{c}{ Agrupamentos } & Médias \\
\hline Trabalhadores & \\
Baixo Conforto & 0,61 \\
Elevada Capacidade de Carga & 0,41 \\
Média Capacidade de Carga & 0,25 \\
Elevado Conforto & 0,24 \\
Baixa Capacidade de Carga & 0,24 \\
26.500,00 & 0,20 \\
29.500,00 & 0,17 \\
Médio Conforto & 0,15 \\
28.000,00 & 0,12 \\
Requintados & \\
Elevado Conforto & 0,63 \\
Baixa Capacidade de Carga & 0,40 \\
Baixo Conforto & 0,27 \\
29.500,00 & 0,23 \\
Elevada Capacidade de Carga & 0,22 \\
Médio Conforto & 0,17 \\
Média Capacidade de Carga & 0,15 \\
26.500,00 & 0,15 \\
28.000,00 & 0,11 \\
Populares & \\
Baixa Capacidade de Carga & 0,56 \\
26.500,00 & 0,37 \\
Elevada Capacidade de Carga & 0,27 \\
Baixo Conforto & 0,23 \\
Média Capacidade de Carga & 0,22 \\
Elevado Conforto & 0,21 \\
29.500,00 & 0,21 \\
28.000,00 & 0,19 \\
Médio Conforto & 0,18 \\
\hline & \\
\hline & \\
\hline
\end{tabular}

Fonte: Elaborado pelo autor

Primeiro Agrupamento (Trabalhadores):

Os resultados mais expressivos para os níveis de atributos foram 'Baixo Nível de Conforto', 'Elevada Capacidade de Carga' e 'Média Capacidade de Carga'. O segmento seria, portanto, representado por indivíduos que buscam um veículo barato, desprovido de conforto, mas com elevada capacidade de carga. Recebe destaque o posicionamento dos três níveis de 'preço' que ficaram com médias baixas na ordem de importância para os atributos.

Segundo Agrupamento (Requintados):

REAd I Porto Alegre - Edição 75 - Nº 2 - maio/agosto 2013 - p. 433-452 
Segmentação a partir das bases específicas não observáveis do produto e o emprego conjunto de técnicas multivariadas: o estudo de caso do veículo multiuso

Destaca-se a média mais alta para todos os agrupamentos: 'Elevado Conforto' $(0,63)$. O nível de atributo que se apresenta a seguir é 'Baixa Capacidade de Carga' e essas duas características já oferecem condições de nomeação do grupo. O atributo preço '29.500,00' posiciona-se na faixa intermediária da ordenação, caracterizando que preço elevado também seria uma opção para a cesta de preferência dos consumidores desse grupo, o mais numeroso com 325 casos.

Terceiro Agrupamento (Populares):

Nesse cluster 'Baixa Capacidade de Carga' recebe a mais alta média do segmento seguido pelo nível mais baixo dentre o atributo 'Preço'. 'Elevado Conforto' e 'Médio Conforto' se apresentam com médias baixas, sugerindo um agrupamento composto por consumidores sensíveis a um veículo básico e econômico.

Interpretando os resultados obtidos com os dois métodos, têm-se alguns pontos comuns no que se refere à composição de comportamento de compra e segmentação de mercado. Com o emprego da técnica de Análise Conjunta de Atributos, constatou-se que a cesta de preferência do consumidor, é criada fundamentalmente em função sequencial do nível de conforto, capacidade de carga e finalmente pelo preço. Analisando a dimensão dos clusters encontrados, tem-se a mesma lógica de importância no tocante a quantidade de indivíduos por atributo (325 indivíduos para conforto, 286 para carga e 189 para preço), obedecendo exatamente a mesma ordem da análise anterior.

\section{CONSIDERAÇÕES FINAIS}

Este estudo objetivou validar a teoria de segmentação de mercado de Green e Krieger (1991) por meio de um estudo de caso, empregando sequencialmente a Análise Conjunta e Análise de Conglomerados. Ao empregar-se o procedimento proposto pelos autores, constatou-se que tal atitude confere robustez ao modelo de identificar atributos e priorizar preferências de consumo. O emprego isolado da Análise Conjunta não conferiu a mesma precisão observada na técnica proposta. $\mathrm{O}$ mesmo poderia ser dito a respeito da Análise de Conglomerados. $\mathrm{O}$ objetivo principal do estudo foi plenamente atingido quando evidenciados resultados semelhantes com o emprego das duas técnicas

Conforme já observado, a segmentação de mercado a partir de bases não observáveis é complexa e multifacetada, questão atenuada pelo emprego das duas técnicas sugeridas. Além REAd I Porto Alegre - Edição 75 - N 2 - maio/agosto 2013 - p. 433-452 
dessa questão, conforme Fávero, Belfiore, da Silva e Chan (2009) a Análise de Conglomerado não é apropriada para inferência de característica da população a partir de dados amostrais. $\mathrm{O}$ estudo apresenta contribuição no sentido de sugerir emprego nas mais diversas situações, destacando redução de dados pela simples redução da informação de uma população ou amostra; criação de hipóteses previamente estabelecidas; classificação de indivíduos bem como objetos; avaliação da utilidade percebida bem como avaliação de valor para fins de segmentação de mercado. Registra-se a ressalva que ambas as técnicas, mesmo que sequencialmente empregadas, caracterizam o procedimento como exploratório por não possuírem base teórica e inferencial, generalizável de amostra para população.

O conceito de veículo multiuso ou minivans é um sucesso no mercado europeu de automóveis, distintamente do que ocorre no Brasil. Na Europa, esse conceito de veículo atende a demanda de pequenas empresas que transportam pequenos volumes e àqueles profissionais liberais de consertos rápidos. No Brasil, talvez em função da baixa renda, o profissional e as pequenas empresas utilizam uma solução mais modesta, composta por um veículo utilitário usado.

Questões como esta frequentemente são observadas pelas montadoras nacionais, entretanto em função de estratégia ou mesmo pelo custo de desenvolvimento de produto, o veículo adota o mesmo conceito, sendo lançado com o mesmo apelo percebido no exterior.

Empregar uma metodologia de identificação de atributos voltada para a segmentação de mercado pode atenuar a questão de reposicionamento do produto, conforme frequentemente se observa quando o desempenho no mercado não corresponde às expectativas.

Como limitação do estudo, destaca-se a questão da falta de estudos correlatos, em outras áreas que efetivamente coloquem em prática a robustez da técnica. Outro aspecto identificado por Green e Krieger (1991) é que o modelo não tem emprego real naqueles produtos tidos como radicalmente novos e sim em recombinações de produtos existentes. Sugere-se, como trabalhos futuros, um estudo de maior amplitude colocando a técnica em situação comprobatória.

\section{REFERÊNCIAS}

AAKER, D. A, V. KUMAR, G. S. DAY. Pesquisa de Marketing. 2ª Tradução: Reynaldo Cavalheiro Marcondes. São Paulo, SP: Atlas, 2004.

REAd I Porto Alegre - Edição 75 - N 2 - maio/agosto 2013 - p. 433-452 
Segmentação a partir das bases específicas não observáveis do produto e o emprego conjunto de técnicas multivariadas: o estudo de caso do veículo multiuso

ALDENDERFER, M. S.; BLASHFIELD, R. K. Cluster Analysis. Beverly Hills, CA: Sage, 1984.

ALLEN, M. A practical method for uncovering the direct and indirect relationships between human values and consumer purchases. Journal of Consumer Marketing, Arvada, v. 18, n. 2, p. 102-117, 2001.

ALLEN, M.; NG, S.; WILSON, M. A functional approach to instrumental and terminal values and the valueattitude. European Journal of Marketing, Birmingham, v.36, n.1;2, p.111-135, 2002.

ALPERT, M. Identification of determinant attributes: a comparison of methods. Journal of Marketing Research, Pennsylvania, v. 8, n. 2, p. 184-191, may 1971.

ASSAEL, H. Consumer Behavior \& Marketing Action, Kent Publishing Co., Boston, 1992.

BITTENCOURT, H. R., e J. M. FACHEL. Análise Conjunta de Atributos (Conjoint Analysis), Porto Alegre, n. 1, p. 1-46, 1999. Documentos de Estudo do PPGA, 1999: 1-46. BORG, I.; GROENEN, P. J. F. Modern Multidimensional Scaling: Theory and Applications. New York: Springer-Verlag. 1997.

BROCHADO, A; MARTINS, F. Aspectos Metodológicos da Segmentação de Mercado: base de segmentação e métodos de classificação. Revista Brasileira de Gestão de Negócios. São Paulo, V. 10, n 27. 132-149-abril-junho 2008.

ENGEL, J. F.; BLACKWELL, R. D.; MINIARD, P. W. Comportamento do consumidor. 8. ed. Rio de Janeiro: Livros Técnicos e Científicos S.A., 2000. Englewood Cliffs, pp. 317-339

EVERITT, B. Cluster Analysis. New York, NY: Haltesd, 1980.

FÁVERO, L. P; BELFIORE, P; DA SILVA, F. L; CHAN, B. L. Modelagem multivariada para tomada de decisões. São Paulo: Editora Campus, 2009. p. 544

FISHBEIN, M. Readings in attitude theory and measurement. New York: John, 1967. GREEN, P. E.; KRIEGER, A. M. Segmenting markets with conjoint analysis. Journal of Marketing, Los Angeles, v.55, n. 4, p.20-31, 1991 
HAIR, J; ANDERSON, R. E.; TATHAM, R. L.; BLACK W. C. Análise Multivariada de Dados. 5. ed. Porto Alegre: Bookman, 2005.

HERRMANN, A. Perception and Preference Formation Patterns of Individuals when Purchasing Brands of Different Product Categories, Journal of International Marketing and Marketing Research, Pennsylvania, vol. 19, nº 2, 59-73, 1994.

HOLBROOK, M.B. What Is Consumer Research? Journal of Consumer Research, Pennsylvania, v. 14, p. 128-132, jun 1987.

KANUK, L.; SCHIFFMAN, L. G. Comportamento do consumidor. 6. ed. Rio de Janeiro: LTC: 2000.

KRUSKAL, J. B. Nonmetric Multidimensional Scaling: A Numerical Method. Psychometrika v. 2, p 115-129. 1964

LUTZ, R. The Role of Attitude Theory in Marketin New Jersey, in KASSARJIAN, H. e ROBERTSON, T. (eds.), Perspectives in Consumer Behavior, Prentice-Hall, 1991

MALHOTRA, N. Pesquisa de Marketing. Porto Alegre: Bookman, 2001.

MENEGHIM, L. AN Veículos. 18 de Novembro de 2001.

http://www1.an.com.br/2001/nov/18/0vei.htm (acesso em 24 de Setembro de 2009).

MORGAN, D. L. Focus groups as qualitative research. Beverly Hills: SAGE

Publications, 1988.

MOTTA, P. C. Análise Conjunta. Rio de Janeiro: PUC - RJ, Mímeo, 1997.

MOWEN, J. C.; MINOR, M. S. Comportamento do consumidor. São Paulo: Prentice Hall, 2008.

NARAYANA, C. L.; MARKIN, R. J. Consumer behavior and product performance: na alternative conceptualization. Journal of Marketing, Los Angeles, v 39, p. 1-6,.oct 1975,

REAd I Porto Alegre - Edição 75 - N 2 - maio/agosto 2013 - p. 433-452 
Segmentação a partir das bases específicas não observáveis do produto e o emprego conjunto de técnicas multivariadas: o estudo de caso do veículo multiuso

NOWLIS, S.M.; SIMONSON, I. Attribute-task compatibility as a determinant of consumer preference reversals. Journal of Marketing Research, Pennsylvania, v. 34, may 1997, 20521

PETER, J. P.; OLSON, J. C. Consumer behavior and marketing strategy. 5. ed. Boston: Irwin/ McGraw-Hill, 1999.

RATCHFORD, B. Cost-benefit models for explaining consumer choice and information seeking behavior. Management Science, v. 28, p. 197-212, Feb. 1982.

ROBINSON, J. The Economics of Imperfect Competition, London: McMillan, 1938

SHAVITT, S. The role of attitude objects in attitude functions. Journal of Experimental Social Psychology, Princeton, v.26, p.124-148, 1990.

SHETH, J. et al. Comportamento do cliente: indo além do comportamento do consumidor. São Paulo: Atlas, 2001

SMITH, W. Product differentiation and market segmentation as alternative marketing strategies. Journal of Marketing, Los Angeles, v 21, p3-8, 1956

SNYDER, M.; DEBONO, K. Appeals to image and claims about quality: undertanding the psychology of advertising. Journal of Personality and Social Psychology, v.49, p.586-597, 1985.

SPIVEY, W.; MUNSON, J. LOCANDER, L. Improving the effectiveness of persuasive communications: Journal of Marketing Research, Pennsylvania, v12, 60-67.

WOODRUFF, R. B.; GARDIAL, S. F. Know your customer: new approaches to understanding customer value and satisfaction. Malden: Blackwell Business, 1996. WRIGHT, P., Consumer choice strategies: simplifying vs optimizing, Journal of Marketing Research, Pennsylvania, v. 12 p.60-7, 1975

WU, T.W.; DAY, R.L. \& MacKAY, D.B. Consumer Benefits Versus Product Attributes: An Experimental Test. Quarterly Journal of Business and Economics, v. 27, p 88-113, 1988.

REAd I Porto Alegre - Edição 75 - Nº 2 - maio/agosto 2013 - p. 433-452 